\title{
DETERMINAÇÃO DA VELOCIDADE DE ARRASTE DE NANOPARTÍCULAS DE FERRITA DE MN-ZN*
}

\author{
Letícia dos Santos Aguilera ${ }^{1}$ \\ Lucas Barbosa Balthazar ${ }^{2}$ \\ José Brant de Campos ${ }^{3}$ \\ Ronaldo Sergio de Biasit \\ André Ben-Hur da Silva Figueiredo ${ }^{5}$
}

\section{Resumo}

Nanopartículas magnéticas são amplamente empregadas em diversas áreas devido as suas propriedades superparamagnéticas, e vem sendo estudadas por todo o mundo com potencial em campos como biomedicina e tratamento de efluentes. Nesse experimento as propriedades de ferritas mistas de manganês e zinco foram testadas e avaliadas com base na sua concentração de zinco em cada composto, usando como fator de comparação suas velocidades médias de arraste em um tanque de ondas graduado na presença de um campo magnético externo. $O$ processo procura viabilizar um método de análise que sugira possível coerência com resultados observados em outro método de caracterização magnética, a magnetometria por amostra vibrante. Tais resultados apresentaram maior magnetização de saturação para as amostras de $\mathrm{Mn}_{0,5} \mathrm{Zn}_{0,5} \mathrm{Fe}_{2} \mathrm{O}_{4}$ assim como foi observado maior velocidade de arraste nas amostras de mesma estequiometria.

Palavras-chave: Nanopartículas magnéticas; Ferritas $\mathrm{Mn}-\mathrm{Zn}$; Velocidade de arraste.

\section{SPEED OF DRAG OF Mn-Zn FERRITE NANOPARTICLES DETERMINATION}

\begin{abstract}
Magnetic nanoparticles are widely used in many areas due to their superparamagnetic properties and are being studied worldwide with potential in fields such as biomedicine and effluent treatment. In this experiment the properties of mixed ferrite of manganese and zinc were tested and evaluated based on their zinc concentration in each compound, using as a comparison factor their average speed of drag in a graduated tank of waves, when it is in the presence of an external magnetic field. The process seeks to make feasible a method of analysis that suggests possible coherence with results observed in another magnetic characterization method, vibrant sample magnetometry. These results presented higher saturation magnetization for the samples of $\mathrm{Mn}_{0.5} \mathrm{Zn}_{0.5} \mathrm{Fe}_{2} \mathrm{O}_{4}$ as well as a higher speed of drag in the samples of the same stoichiometry.
\end{abstract}

Keywords: Magnetic nanoparticles; Mn-Zn ferrites; Speed of drag.

1 Bacharel em Engenheira de Petróleo, Mestra em Ciência dos Materiais, Doutoranda em Ciência dos Materiais, Seção de Engenharia Mecânica e de Materiais, Instituto Militar de Engenharia, Rio de Janeiro, RJ, Brasil.

2 Graduando em Engenharia de Materiais, Curso Básico, Instituto Militar de Engenharia, Rio de Janeiro, RJ, Brasil.

3 Bacharel em Engenharia Metalúrgica, Mestre em Engenharia Metalúrgica, Doutor em Engenharia Metalúrgica, Professor, Departamento de Engenharia Mecânica, Universidade do Estado do Rio de Janeiro, Rio de Janeiro, RJ, Brasil. 
4 Bacharel em Engenharia Elétrica, Mestre em Engenharia Elétrica, Ph.D. em Engenharia Elétrica Professor Emérito Seção de Engenharia Mecânica e de Materiais, Instituto Militar de Engenharia, Rio de Janeiro, RJ, Brasil

5 Bacharel em Física, Mestre em Engenharia Nuclear, Doutor em Ciência dos Materiais, Professor, Curso Básico e Seção de Engenharia Mecânica e de Materiais, Instituto Militar de Engenharia, Rio de Janeiro, RJ, Brasil. 


\section{INTRODUÇÃO}

A procura por materiais alternativos para aplicações especializadas em diversos casos cresce a cada dia com o aparecimento de novos problemas com processos atuais. As ferritas tem tido seu papel assegurado nas demandas atuais graças às suas propriedades superparamagnéticas quando seu tamanho é reduzido (nanopartícula), tendo aplicações diretas nos mais vastos campos de pesquisa.

O processo aqui estudado teve como objetivo buscar uma forma de substituir métodos de maior custo, como a magnetometria por amostra vibrante e magnetometria por dispositivo de interferência quântica (SQUID), na obtenção de resultados criando oportunidade de posteriores estudos mais avançados no ramo, possibilitando a geração de conclusões mais rápidas, baratas e ao mesmo tempo pertinentes. Para isso, a velocidade de arraste de ferritas mistas $\mathrm{Mn}$ - $\mathrm{Zn}$ foi observada para três concentrações de zinco distintas quando dispersas em parafina em presença de um campo magnético externo aplicado.

Trata-se de um estudo cujos parâmetros utilizados foram baseados em outros trabalhos [1,2], tendo variações simplificadas no intuito de agregar conhecimento para futuras continuidades nas pesquisas do comportamento magnético das nanopartículas, seguindo os conceitos consolidados para as ferritas em dimensões maiores.

Todos os materiais possuem propriedades magnéticas, porém só é possível classificá-las na presença de um campo magnético. As nanopartículas magnéticas aqui estudadas apresentam comportamento superparamagnético que, acima da temperatura de bloqueio, estabelece que o material só se magnetize quando exposto a um campo magnético externo, e após a remoção desse o campo não apresenta magnetização remanescente.

A estrutura cristalina das ferritas do tipo espinélio $\left(\mathrm{MFe}_{2} \mathrm{O}_{4}\right.$, em que $\mathrm{M}$ é um metal divalente) é formada por um empacotamento cúbico de face centrada (CFC) de átomos de oxigênio de maneira que os íons metálicos ocupam os interstícios entre esses átomos, denominados sítios tetraédricos (localizados entre 4 íons de oxigênio) e sítios octaédricos (localizados entre 6 íons de oxigênio) e a ocupação dos íons divalentes nos sítios vai dar origem ao comportamento magnético do material [3].

Ferritas formadas por mais de um metal divalente são chamadas de ferritas mistas. Neste trabalho, a ferrita mista de Mn-Zn foi o objeto de estudo.

Ferritas em dimensões não nanométricas, denominadas bulk tem suas propriedades alteradas quando o íon não magnético de zinco é inserido na sua estrutura [4]. Observa-se uma diferenciação na interação dos íons que ocupam os sítios octaédricos e tetraédricos gerando um momento magnético de spin resultante não nulo até que, para uma concentração grande de zinco, haja a predominância das propriedades paramagnéticas do íon de zinco.

Neste sentido, a velocidade de arraste das nanopartículas verificada para três diferentes concentrações de zinco sugere uma mesma tendência de variação que a magnetização em função da concentração de zinco apresentada na literatura para ferritas bulk, como apresentada na Figura 1. 


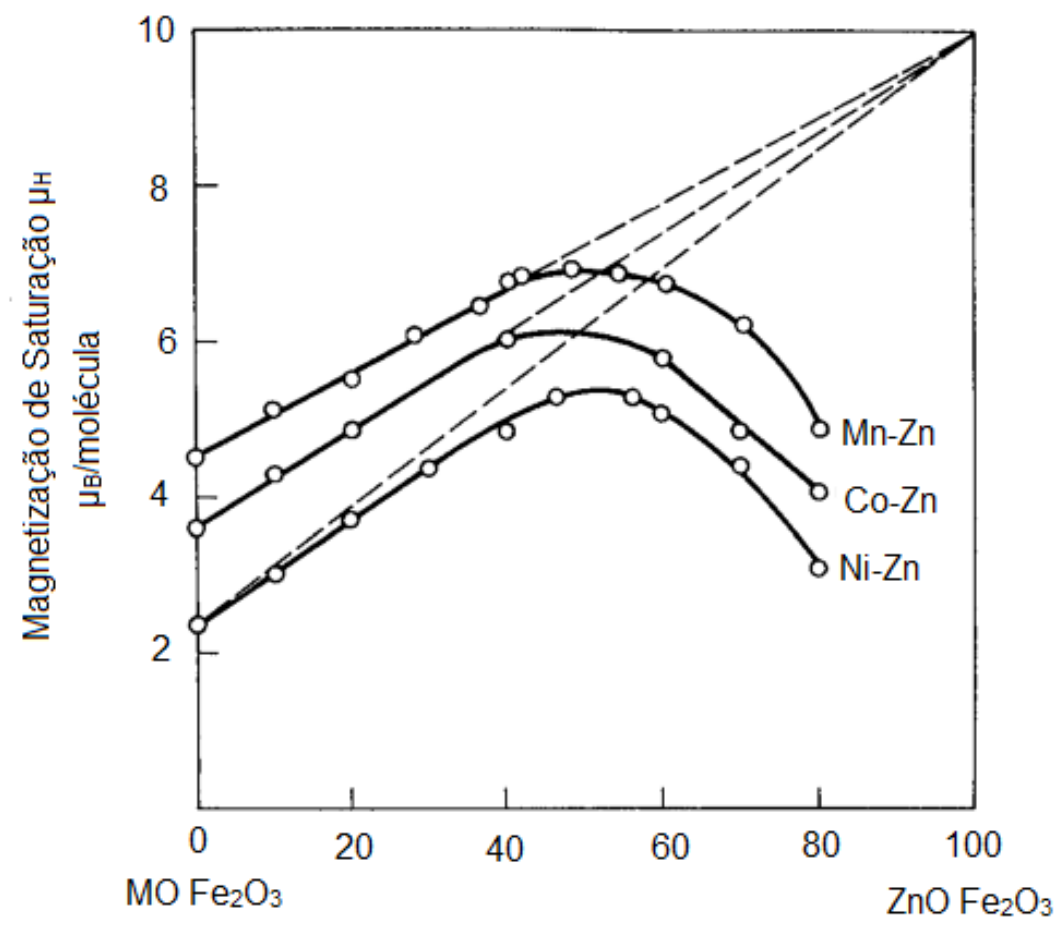

Porcentagem em mol de $\mathrm{ZnO} \mathrm{Fe} 2 \mathrm{O}_{3}$

Figura 1 Efeito da Magnetização de Saturação (a OK) pela adição de Zn nas ferritas de Mn, Co e Ni [4].

\section{MATERIAIS E MÉTODOS}

O experimento se consistiu na medição da velocidade de arraste da ferrita mista, em três diferentes concentrações de zinco dispersas em matriz de parafina, na direção e sentido de um campo magnético externo aplicado a uma determinada distância. A partir disso, a curva de velocidade média de arraste por concentração de zinco foi obtida para o material. Tais resultados foram comparados com a curva de magnetização de saturação por concentração de zinco obtida a partir da análise de magnetometria por amostra vibrante.

\subsection{Síntese por Combustão em Solução (SCS)}

A fabricação da ferrita mista se deu pela utilização do método de síntese por combustão em solução na qual nitratos metálicos são misturados a um combustível e colocados em solução em água destilada. A solução é aquecida a $100^{\circ} \mathrm{C}$ até que entre em combustão autossustentada e haja a formação de uma espuma facilmente desaglomerável e, após maceração mecânica manual, o pó de nanopartículas é obtido.

Foram sintetizadas nanopartículas das seguintes ferritas: $\mathrm{MnFe}_{2} \mathrm{O}_{4}$, $\mathrm{Mn}_{0,50} \mathrm{Zn}_{0,50} \mathrm{Fe}_{2} \mathrm{O}_{4}$ e $\mathrm{ZnFe}_{2} \mathrm{O}_{4}$, respeitando a estequiometria para a formação das soluções dos nitratos metálicos e a glicina (combustível utilizado), na razão GlicinaNitrato igual a 1. 


\subsection{Preparação dos Corpos de Prova}

O pó de nanopartículas obtido da síntese foi disperso em parafina liquefeita a $60^{\circ} \mathrm{C}$ em chapa aquecedora formando um compósito, na proporção 1:10. Esse compósito liquefeito foi colocado em matriz metálica cilíndrica de $15 \mathrm{~mm}$ de diâmetro interno e $37 \mathrm{~mm}$ de comprimento. Abaixo de $36^{\circ} \mathrm{C}$, a parafina solidifica e então um bloco sólido é formado. A partir disso, corpos de prova entre $5,0 \mathrm{~mm}$ e $6,5 \mathrm{~mm}$ de altura são obtidos para o ensaio de arraste.

Esse processo foi repetido para cada uma das ferritas em todas as concentrações de zinco.

\subsection{Método de Arraste}

O método trata da medição da velocidade média de deslocamento dos corpos de prova em direção ao campo magnético fixo aplicado. O deslocamento foi feito em um tanque de ondas de $2500 \mathrm{~cm}^{2}$ com escala graduada, preenchido com 2 litros de água destilada.

O corpo de prova é colocado a $10 \mathrm{~cm}$ de distância do imã e a velocidade é determinada pela razão entre essa distância percorrida e o tempo gasto para 0 deslocamento.

Cada corpo de prova foi ensaiado 3 vezes, sendo 4 corpos de prova para cada ferrita sintetizada. Dessa forma, a velocidade média de arraste foi obtida por 12 velocidades de arraste medidas.

\subsection{Magnetometria por Amostra Vibrante}

O comportamento magnético das nanopartículas dispersas em parafina foi investigado pela técnica de magnetometria por amostra vibrante através do ensaio dos corpos de prova fabricados de cada concentração de zinco no magnetômetro Princeton Appllied Research modelo 155, com campo aplicado de $9 \mathrm{kOe}$ em temperatura ambiente, no Laboratório de Magnetometria do Centro Brasileiro de Pesquisas Físicas (CBPF).

\section{RESULTADOS E DISCUSSÃO}

\subsection{Método de Arraste}

$\mathrm{Na}$ Tabela 1 são apresentados os resultados da velocidade média dos corpos de prova para as nanopartículas de ferritas de $\mathrm{MnFe}_{2} \mathrm{O}_{4}, \mathrm{Mn}_{0,50} \mathrm{Zn}_{0,50} \mathrm{Fe}_{2} \mathrm{O}_{4}$ e $\mathrm{ZnFe}_{2} \mathrm{O}_{4}$. A partir desses dados, a curva apresentada na Figura 2 foi traçada considerando a tendência dos dados nos intervalos das concentrações. 
Tabela 1: Resultados do ensaio de arraste para as nanopartículas de $\mathrm{MnFe}_{2} \mathrm{O}_{4}, \mathrm{Mn}_{0,50} \mathrm{Zn}_{0,50} \mathrm{Fe}_{2} \mathrm{O}_{4} \mathrm{e}$ $\mathrm{ZnFe}_{2} \mathrm{O}_{4}$

\begin{tabular}{|c|c|c|c|}
\hline Ferrita & Tempo (s) & Distância (m) & Velocidade (m/s) \\
\hline $\mathrm{MnFe}_{2} \mathrm{O}_{4}$ & 13,72 & 0,01 & 0,000729 \\
\hline $\mathrm{Mn}_{0,50} \mathrm{Zn}_{0,50} \mathrm{Fe}_{2} \mathrm{O}_{4}$ & 13,69 & 0,01 & 0,000732 \\
\hline $\mathrm{ZnFe}_{2} \mathrm{O}_{4}$ & 49,61 & 0,01 & 0,000202 \\
\hline
\end{tabular}

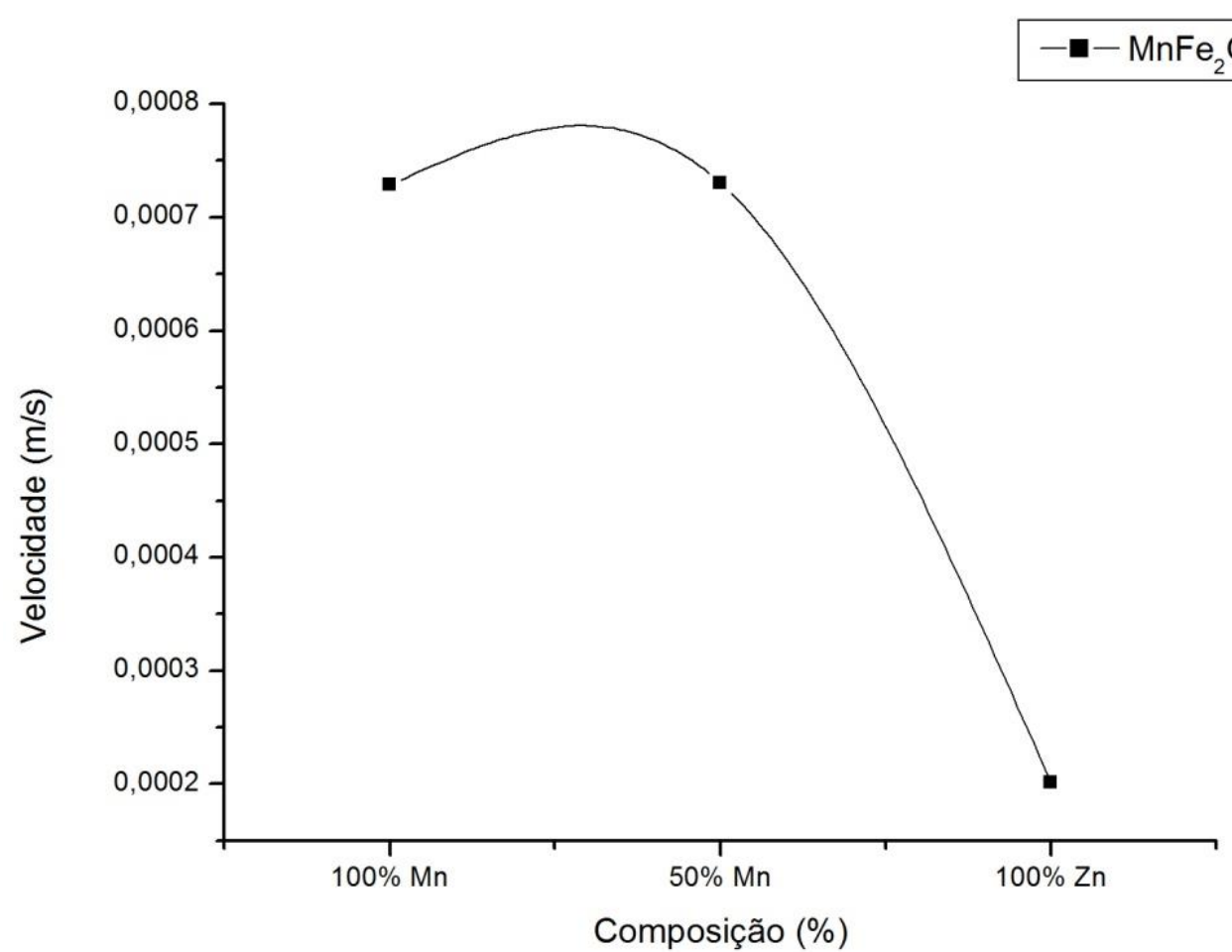

Figura 2 Velocidade de arraste pela concentração de zinco para as nanopartículas de $\mathrm{MnFe}_{2} \mathrm{O}_{4}$, $\mathrm{Mn}_{0,50} \mathrm{Zn}_{0,50} \mathrm{Fe}_{2} \mathrm{O}_{4}$ e $\mathrm{ZnFe}_{2} \mathrm{O}_{4}$

\subsection{Magnetometria por Amostra Vibrante}

A curva de magnetização por campo magnético para as amostras é mostrada na Figura 3. A variação da magnetização de saturação pela concentração de zinco é apresentada na Figura 4. As curvas apresentaram histerese desprezível, comportamento esperado para materiais superparamagnéticos de acordo com a literatura [5] e verifica-se uma maior magnetização para as nanopartículas de ferrita $\mathrm{Mn}_{0,50} \mathrm{Zn}_{0,50} \mathrm{Fe}_{2} \mathrm{O}_{4}$. 


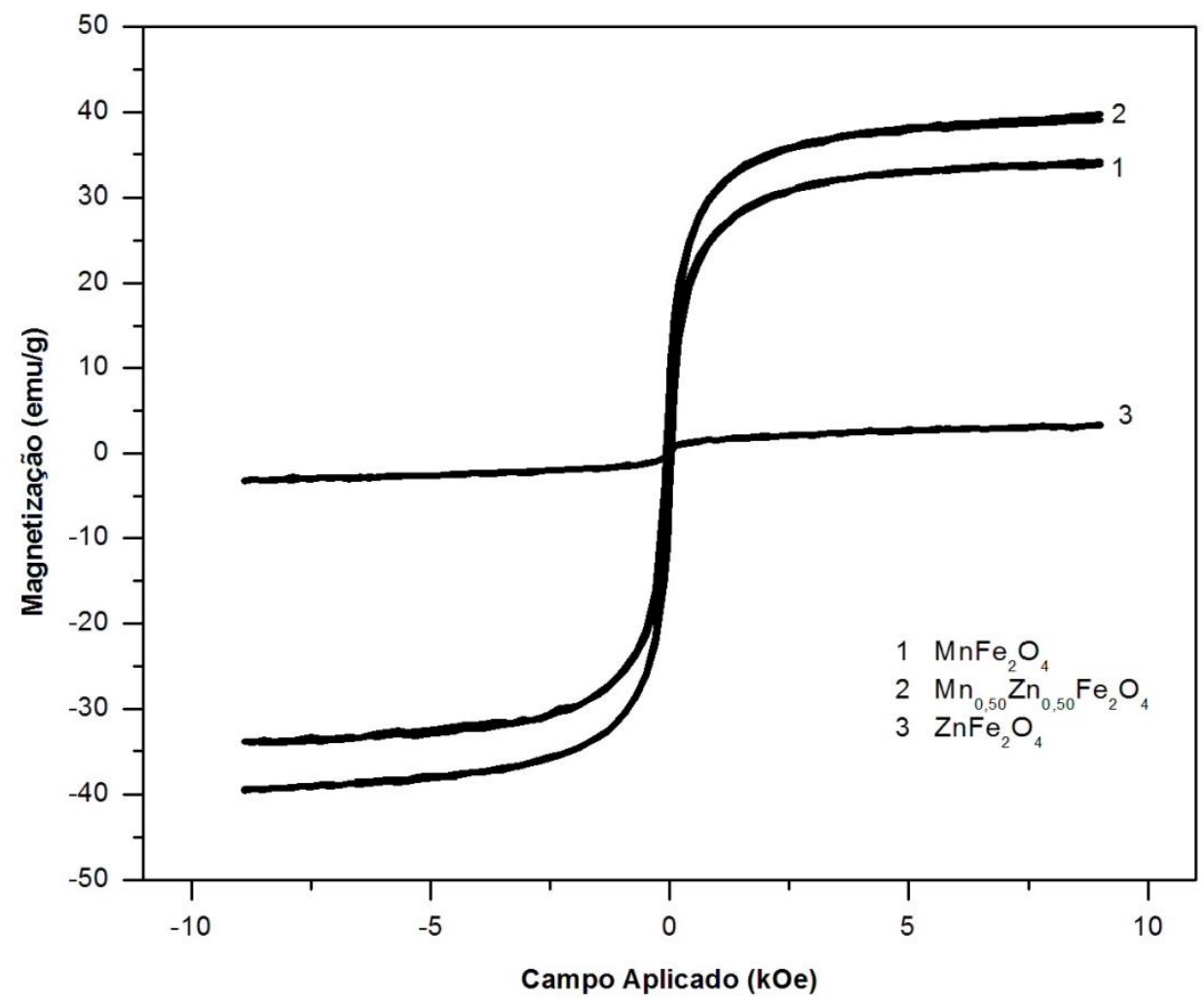

Figura 3 Magnetização das ferritas de Mn-Zn em matriz de parafina

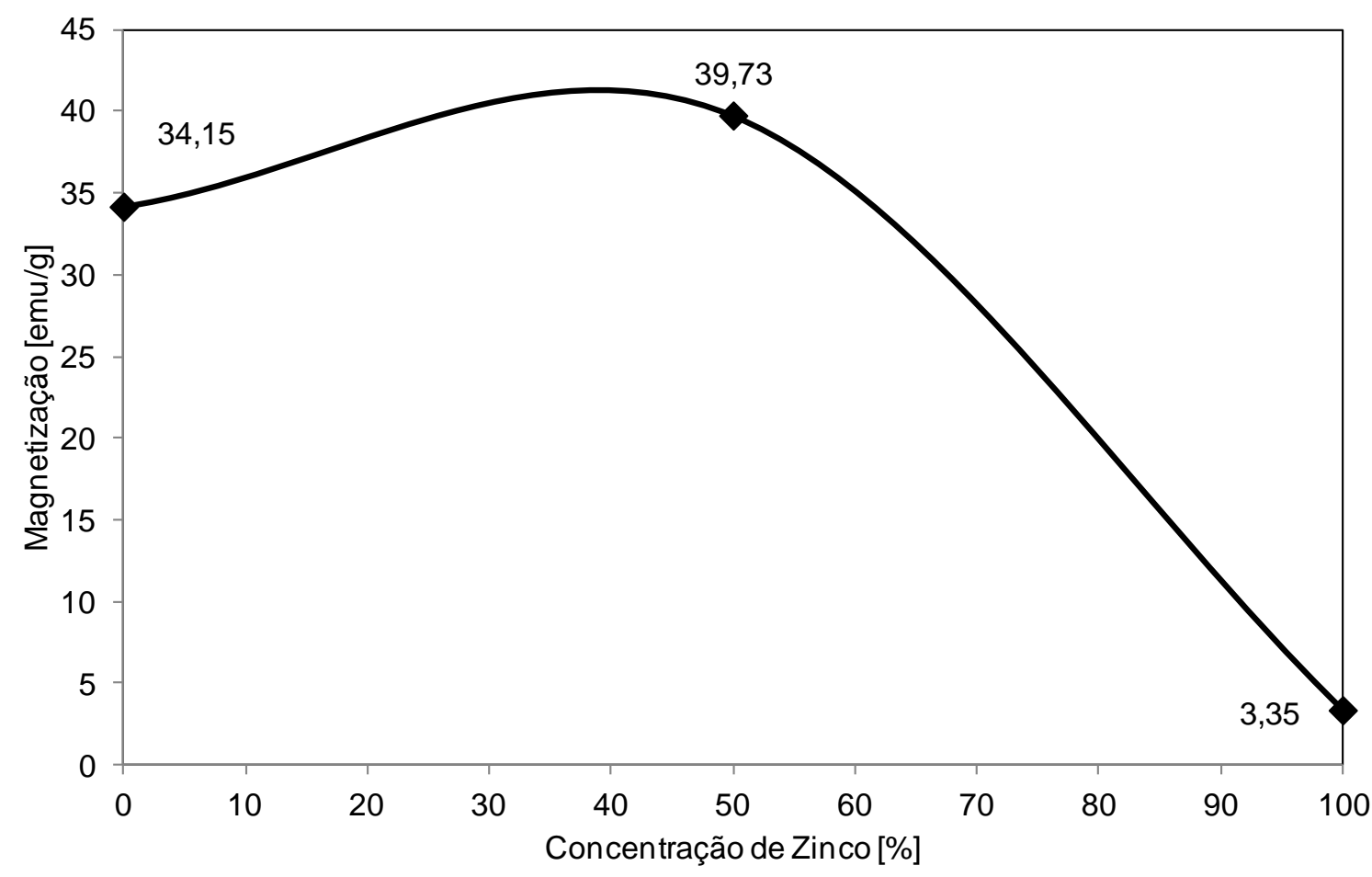

Figura 4 Magnetização de saturação por concentração de zinco 
É possível verificar que a curva de magnetização por concentração de zinco apresenta a mesma tendência que a curva de velocidade de arraste por concentração de zinco obtida pelos dados do método de arraste, apresentada na Figura 2. Essa tendência sugere que seja possível estimar o comportamento magnético das ferritas mistas Mn-Zn pelo método de arraste. Além disso, as curvas são muito semelhantes àquelas apresentadas em [4] para as ferritas bulk, que mesmo o íon de zinco não sendo magnético, quando inserido na estrutura da ferrita que contenha um metal divalente magnético gera um momento magnético de spin resultante maior em concentrações próximas a $50 \%$.

\section{CONCLUSÃO}

Diante dos resultados obtidos, é possível dizer que o método de arraste se mostrou uma ferramenta interessante para a verificação do comportamento magnético da ferrita mista de $\mathrm{Mn}$ - $\mathrm{Zn}$ sintetizada pelo método de combustão em solução, já que a curva adquirida a partir das velocidades médias de arraste dos corpos de prova pela concentração de zinco tem a mesma tendência daquela apresentada na literatura para ferritas bulk e da curva de magnetização por concentração de zinco obtida pela técnica de magnetometria por amostra vibrante.

Tais resultados sugerem que é possível, através de mais estudos, estimar o comportamento magnético das nanopartículas em substituição aos métodos usuais de caracterização magnética, como a própria magnetometria por amostra vibrante e a magnetometria por dispositivo de interferência quântica.

O método de arraste se vale de outra importante vantagem em relação aos métodos usuais: baixo custo, fácil execução e não requer espaços físicos extensos ou salas dedicadas ao método, além de ser de simples reprodutibilidade.

\section{Agradecimentos}

Os autores agradecem à CAPES e ao CNPq pelo apoio financeiro.

\section{REFERÊNCIAS}

[1] Aguilera, L. S. Método de Arraste utilizado para a determinação do comportamento magnético de nanopartículas de ferrita mista de Mn-Zn. 201669 p. Dissertação (Mestrado em Ciência dos Materiais) - Instituto Militar de Engenharia, Rio de Janeiro, 2016.

[2] Cortopassi, W. A., Monteiro, A. P., Braz, A. S. A., Santos, E. H., De Abreu, A. C. A. F., Oliveira, F. A., Carvalhosa, J. C., De Oliveira, L. R. M., De Oliveira, F. M., De Oliveira, B. S. V., Figueiredo, A. B. S. Uso de nanopartículas de ferrita de cobalto para a remoção de óleo na superfície da água com turbilhonamento. Revista Militar de Ciência e Tecnologia. v. XXVIII - p. 24-32. 1o trimestre de 2011.

[3] Cardoso, L. H. G. Nanopartículas Magnéticas de Ferritas Mistas de Cobalto e Zinco. 2011. 103 p. Tese (Doutorado em Ciência dos Materiais) - Instituto Militar de Engenharia, Rio de Janeiro, 2011. 
[4] Cullity, B. D. e Graham, C. D. Introduction to Magnetic Materials, 2nd edition. Wiley, New Jersey, 550 p., 2009.

[5] ARULMURUGAN, R. et. al. Mn-Zn ferrite nanoparticles for ferrofluid preparation: Study on thermal-magnetic properties. Journal Of Magnetism And Magnetic Materials, v. 298, n. 2, p.83-94, mar. 2006. Elsevier BV. http://dx.doi.org/10.1016/j.jmmm.2005.03.002. 